\title{
Baicalin administration attenuates hyperglycemia-induced malformation of cardiovascular system
}

\author{
Guang Wang ${ }^{1,2,3}$, Jianxin Liang ${ }^{1,2}$, Lin-rui Gao ${ }^{1}$, Zhen-peng Si ${ }^{4}$, Xiao-tan Zhang ${ }^{1}$, Guo Liang ${ }^{1}$, Yu Yan ${ }^{1}$, Ke Li', \\ Xin Cheng ${ }^{1}$, Yongping Bao ${ }^{5}$, Manli Chuai ${ }^{6}$, Li-guo Chen², Da-xiang $\mathrm{Lu}^{7}$ and Xuesong Yang ${ }^{1,3}$
}

\begin{abstract}
In this study, the effects of Baicalin on the hyperglycemia-induced cardiovascular malformation during embryo development were investigated. Using early chick embryos, an optimal concentration of Baicalin (6 $\mu \mathrm{M})$ was identified which could prevent hyperglycemia-induced cardiovascular malformation of embryos. Hyperglycemia-enhanced cell apoptosis was reduced in embryos and HUVECs in the presence of Baicalin. Hyperglycemia-induced excessive ROS production was inhibited when Baicalin was administered. Analyses of SOD, GSH-Px, MQAE and GABAA suggested Baicalin plays an antioxidant role in chick embryos possibly through suppression of outwardly rectifying $\mathrm{Cl}(-)$ in the high-glucose microenvironment. In addition, hyperglycemia-enhanced autophagy fell in the presence of Baicalin, through affecting the ubiquitin of p62 and accelerating autophagy flux. Both Baicalin and Vitamin C could decrease apoptosis, but CQ did not, suggesting autophagy to be a protective function on the cell survival. In mice, Baicalin reduced the elevated blood glucose level caused by streptozotocin (STZ). Taken together, these data suggest that hyperglycemia-induced embryonic cardiovascular malformation can be attenuated by Baicalin administration through suppressing the excessive production of ROS and autophagy. Baicalin could be a potential candidate drug for women suffering from gestational diabetes mellitus.
\end{abstract}

\section{Introduction}

Roots of Scutellaria baicalensis are used in Chinese medicine and it reputedly calms fetuses in pregnant women $^{1,2}$. Baicalin, a flavone glycoside (the glucuronide of Baicalein), is formed via the binding of glucuronic acid with Baicalein, in the roots of Scutellaria baicalensis and Scutellaria lateriflora. Baicalin is principally used in Asian countries as a herbal supplement because of its wide variety of health benefits, including anti-neuroinflammatory ${ }^{3}$, anticancer $^{4}$ and anti-anxiety effects ${ }^{5}$. It can also improve lung

Correspondence: Xuesong Yang (yang_xuesong@126.com)

'Division of Histology \& Embryology, Joint Laboratory for Embryonic Development \& Prenatal Medicine, Medical College, Jinan University, Guangzhou 510632, China

${ }^{2}$ Chinese Medicine College, Jinan University, Guangzhou 510632, China Full list of author information is available at the end of the article

These authors contributed equally: Guang Wang, Jianxin Liang.

Edited by A. Finazzi-Agró function $^{6}$ and fertility ${ }^{7}$.The most common mechanism of Baicalin being against pathogenesis is its protective effect by reducing the production of oxidative stress ${ }^{8}$. Interestingly, Qi et al. ${ }^{9}$ reported that Baicalin could enhance the developmental competence of mouse embryos through suppressing cellular apoptosis and HSP70 expression, and activating DNA methylation. However, whether or not Baicalin can prevent malformation of the cardiovascular system caused by environmental hazards at an early stage remains unclear. This study focuses on the potential beneficial effects of Baicalin on the early development of heart tube and vasculature, in order to explore whether or not Baicalin could be a potential candidate drug for pregnant women.

During embryonic development, the cardiovascular system appears first, since oxygen and nutrients need to 
be delivered to the tissues and waste products must be removed through blood circulation as the embryo develops. The cardiovascular system is composed of heart and blood vessels, including arteries, veins and capillaries in the adult. Heart and vasculature derive from both the embryonic mesoderm and extra-embryonic yolk sac. During early embryonic development, the heart initially forms in embryonic disc as a simple paired heart tube inside the forming pericardial cavity. During the process, the heart formation undergoes a series of transformations. A straight heart tube derives from the fusion of bilateral cardiomyocytes in primary/secondary heart fields at embryonic midline ${ }^{10}$, and then followed by the right looping of the heart tube and sepetation ${ }^{11}$. Endodermderived signals such as bone morphogenetic protein (BMP), fibroblast growth factor and Wnt antagonist are indispensable for precardiac mesoderm cells to differentiate into mature cardiomyocytes during cardiomyogenesis $^{12-14}$. Moreover, Nkx2.5 and GATA factors are all considered to be the important cardiogenic transcription factors that characterize and induce cardiogenic differentiation ${ }^{15}$.

Meanwhile, vasculature formation occurs in embryogenesis at three different morphological stages, including vasculogenesis, angiogenesis and vascular remodeling. Both vasculogenesis and angiogenesis are accomplished in the prenatal period. Vasculogenesis depicts the formation of initial primitive vascular plexus derived from hemangioblasts in extra-embryonic yolk sacs or within the embryo. The earliest appearance is in blood islands located in the extra-embryonic region, in which the mesoderm contributes to blood islands and then these cells differentiate into both the epithelium of blood vessels and fetal red blood cells. Morphologically, these blood islands join together to form the primary blood plexus (vasculogenesis) that connects with the developing heart tube to form the integrated cardiovascular system. Angiogenesis involves the remodeling and expansion of the vascular plexus through endothelial sprouting and intussusceptive microvascular growth ${ }^{16}$. The yolk sac membrane (YSM) in the extra-embryonic region is the initial site where blood vessels and angioblasts develop, and the physiological function of YSM is to provide nutrition to the developing embryo. The chick chorioallantoic membrane (CAM) is a highly vascularized membrane underneath the inner surface of the eggshell, and CAM is formed by the fusion of the chorionic membrane and allantois during embryo development. Both chick YSM and CAM are excellent in vivo models for studying the angiogenesis because they can be easily accessed and manipulated in vitro ${ }^{17-20}$.

Gestational diabetes is due to glucose intolerance during pregnancy and presents as high blood glucose levels at the beginning of pregnancy. The incidence of congenital malformations in diabetic pregnancy has been reported to be $2-5$ times higher than in non-diabetic pregnancy ${ }^{21}$. Dysplasia of the cardiovascular system during cardiogenesis and angiogenesis leads to constantly impaired fetal development or death. However, there is no effective drug for the protection of embryonic development of gestational diabetes mellitus. In our previous studies, early gastrulating chick embryo was employed as an experimental model in gestational diabetes-induced cardiovascular malformation ${ }^{22,23}$. We found both reactive oxygen species (ROS) and autophagy play an important role in chick embryo malformation. In the present study, Baicalin was shown to exhibit a protective effect on early cardiovascular development, and the underlying mechanisms were partly dissected using in vivo chick and mouse models, and HUVEC cell line.

\section{Materials and methods \\ Mice and treatment \\ Reagents}

Baicalin (99\% purity) was purchased from Santa Cruz Biotechnology (Dallas, TX, USA). According to the results of Chen et al., $40 \mathrm{mg} / \mathrm{kg}$ body weight (BW) of Baicalin can alleviate the effect of mifepristone on the levels of serum estrogen/progesterone and the important molecules of canonical Wnt signaling pathway during periimplantation period in mice ${ }^{24}$.

\section{Experimental animal groups and treatments}

Kunming mice were obtained from the Laboratory Animal Centre of Sun Yat-sen University (Guangzhou, China). Diabetes mellitus was induced in 8-week-old female mice by injecting streptozotocin (STZ; Sigma, St. Louis, MO, USA; dissolved in $0.01 \mathrm{~mol} / \mathrm{l}$ citrate buffer, $\mathrm{pH} 4.5$ ) at 75 $\mathrm{mg} / \mathrm{kg} \mathrm{BW}$ for three consecutive days. Blood glucose levels were measured by the Roche Accu-Chek Aviva Blood Glucose System (Roche, Penzberg, BY, Germany) for 7 days after STZ injection. Diabetes mellitus was characterized as non-fasting blood glucose level exceeding $16 \mathrm{mM}^{16,17}$. Mice with diabetes mellitus were randomly divided into two groups: a $40 \mathrm{mg} / \mathrm{kg}$ BW Baicalin treatment group $(n=$ $12)$ and a physiological saline treatment group $(n=11)$. In control $(n=6)$ and diabetes mellitus groups, the mice were administered with $0.1 \%$ dimethyl sulfoxide solution via an intra-gastric gavage for 1 week. The mice in Baicalin group were administered with $1 \mathrm{ml} /$ day of Baicalin by intragastric gavage for 1 week.

This study was carried out in strict accordance with the recommendations of the Guide for the Care and Use of Laboratory Animals of the National Institutes of Health. The protocol was approved by the Committee on the Ethics of Animal Experiments of the Jinan University. All surgeries were performed under pentobarbital anesthesia, and all efforts were employed to minimize suffering. 


\section{Avian embryos and treatment}

Fertilized chick eggs were obtained from the Avian Farm of the South China Agriculture University. The eggs were incubated until the required stage ${ }^{25}$ in a humidified incubator (Yiheng Instrument, Shanghai, China) at $38^{\circ} \mathrm{C}$ and $70 \%$ humidity. For early gastrula embryos, $\mathrm{HHO}$ (Hamburger and Hamilton stage) ${ }^{25}$ chick embryos were prepared and incubated in the absence/presence of Baicalin (Sigma, USA) and/or high glucose (Sigma, USA) and Rapa (LC Labs, USA) using early chick culture (EC culture) as described previously ${ }^{26}$.

The embryos were harvested at the desired time based on the experimental requirements after incubation at $38^{\circ} \mathrm{C}$. All of the embryos were photographed using a stereomicroscope (Olympus MVX10, Japan) before being fixed with $4 \%$ paraformaldehyde for morphological and gene expression analysis. Only the surviving embryos were used for further study. For the histological analysis, the treated embryos or yolk sacs were dehydrated, embedded in paraffin wax and serially sectioned at $5 \mu \mathrm{m}$ using a microtome (Leica RM2126RT, Germany). The sections were de-waxed in xylene, rehydrated and stained with either hematoxylin and eosin dye or immunofluorescent stain, and photographed using a fluorescent microscope (Olympus IX50) with the NIS-Elements F3.2 software package.

\section{Assessment of angiogenesis on chick embryonic YSM}

Briefly, the eggs were treated with simple saline, $100 \mu \mathrm{l}$ of $3.75 \mathrm{mg} / \mathrm{ml} \quad 2,2^{\prime}$-Azobis(2-methylpropionamidine) dihydrochloride (AAPH) or/and $6 \mu \mathrm{M}$ Baicalin per $\operatorname{egg}^{18}$. The solutions were directly injected into the blunt air chamber of the fertilized egg and then the embryos were harvested after a further 4.5 days of incubation at $38^{\circ} \mathrm{C}$. All of the harvested YSMs were photographed using a stereomicroscope (Olympus MVX10, Tokyo, Japan) before being used for molecular analyses.

\section{Assessment of angiogenesis using chick CAM}

As described previously ${ }^{27}$, chick embryos were incubated until day 9 when the CAM was well developed. The embryos were treated with $100 \mu \mathrm{l}$ of simple saline, high glucose $(50 \mathrm{mM})$ and/or Baicalin $(6 \mu \mathrm{M})$ for $48 \mathrm{~h}$ and all surviving embryos were then harvested for analysis. The CAM and accompanying blood vessels in the control and high glucose $(50 \mathrm{mM})$ and/or Baicalin $(6 \mu \mathrm{M})$-treated embryos were photographed using a Canon Powershot SX130 IS digital camera (12.1 M Pixels).

\section{Cell lines and culture}

Human umbilical vascular endothelial cells (HUVECs), a gift from Zhi Huang's laboratory, were cultured in Dulbecco's modified Eagle's medium (DMEM) (Gibco,
Shanghai, China) supplemented with $10 \%$ fetal bovine serum (FBS), and incubated at $37^{\circ} \mathrm{C}$ and $5 \% \mathrm{CO}_{2}$.

\section{Histology}

Briefly, 12-week-old mouse livers, mouse kidney (control, STZ-induced-diabetes mellitus, and STZ-induceddiabetes mellitus with Baicalin treatment) were fixed in $4 \%$ paraformaldehyde at $4{ }^{\circ} \mathrm{C}$ for $24 \mathrm{~h}$. The specimens were then dehydrated, cleared in xylene and embedded in paraffin wax. The embedded specimens were serially sectioned at $5 \mu \mathrm{m}$ using a rotary microtome (Leica, RM2126RT, Wetzlar, Hessen, Germany). The sections were stained with hematoxylin and eosin (H\&E), periodic acid Schiff (PAS) reaction, Masson's trichrome dyes (Masson staining), Sirius red or immunohistochemically. The Masson and Sirius red stains were used to reveal the presence of fibrosis in liver sections and the histological alterations of mice's kidney. Photographs were captured of the stained histological sections using an epifluorescence microscope (Olympus IX51, Leica DM 4000B).

\section{Immunofluorescent staining and F-actin/Hoechst/PI staining}

Chick embryos were harvested after a given time incubation and fixed in $4 \%$ PFA overnight at $4{ }^{\circ} \mathrm{C}$. Wholemount embryo immunostaining was performed using the following antibody: MF-20 (1:500, DSHB, USA). Briefly, the fixed embryos were then incubated with this primary antibody at $4{ }^{\circ} \mathrm{C}$ overnight on a shaker. Following extensive washing, the embryos were incubated with anti-rabbit IgG conjugated to Alexa Fluor 488 overnight at $4{ }^{\circ} \mathrm{C}$ on a rocker. For F-actin detection, the cultured cells were stained using phalloidin-Alexa-Fluor 488 (1:200, Invitrogen, USA) at room temperature for $2 \mathrm{~h}$. All the embryos were later counterstained with 4',6-diamidino-2-phenylindole (DAPI; 1:1000, Invitrogen, USA) at room temperature for $1 \mathrm{~h}$. For Hoechst (1:1000, Sigma, USA)/ propidium iodide (PI, 1:1000, Sigma, USA) staining, the cells were cultured and washed twice with cold phosphate-buffered saline (PBS), and then incubated with Hoechst/PI for $45 \mathrm{~min}$ at $37^{\circ} \mathrm{C}$ in the dark.

\section{Dihydroethidium and CCK8 assays}

Dihydroethidium (DHE) staining was performed at the end of cell culture using DHE fluorescent probe (Beyotime, Shanghai, China) to detect the presence of superoxide anion $\left(\mathrm{O}_{2}{ }^{-}\right)$. The cells were incubated with $10 \mu \mathrm{M}$ DHE for $30 \mathrm{~min}$, at $37^{\circ} \mathrm{C}$, and then collected for analyses according to the manufacturer's instructions. HUVECs (control, $6 \mu \mathrm{M}$ Baicalin, $50 \mathrm{mM}$ glucose and $6 \mu \mathrm{M}$ Baicalin $+50 \mathrm{mM}$ glucose group) were seeded into 96 -well plates. These cells $\left(1 \times 10^{6}\right.$ cells $\left./ \mathrm{ml}\right)$ were maintained in DMEM $+10 \%$ fetal bovine serum at $37^{\circ} \mathrm{C}$ and $5 \% \mathrm{CO}_{2}$. The cell 
viability was assessed using CCK8 assay (cholecystokinin8). Briefly, $10 \mu \mathrm{l}$ of CCK8 reagent (Dojindo, Kumamoto, Japan) was added to the 96-well plates and incubated continually for $6,12,24$ and $48 \mathrm{~h}$ at $37^{\circ} \mathrm{C}$. The absorbance values were measured at $450 \mathrm{~nm}$ using a Bio-Rad model 450 microplate reader (Bio-Rad, Hercules, CA, USA). The cell viability was indirectly determined by examining the ratio of the absorbance value of Baicalin or/and glucosetreated cells relative to the control cells, from this experiments.

\section{Western blot}

Chick embryos (HH10) were collected and lysed with CytoBuster $^{\mathrm{TM}}$ Protein Extraction Reagent (\#71009, Novagen). The total protein concentration was determined using a BCA quantification kit (BCA01, DingGuo BioTECH, CHN). Samples containing equal amounts of protein were resolved by odium dodecyl sulfate-polyacrylamide gel electrophoresis and then transferred to polyvinylidene difluoride membranes (Bio$\mathrm{Rad})$. The membranes were blocked with $5 \%$ Difco $^{\mathrm{TM}}$ skimmed milk (BD) and then incubated with primary and secondary antibodies. The antibodies used were GATA4 (Bioworld, USA); LC3B (Cell Signaling Technology, USA); Beclin1 (Bioworld, USA); C-Caspase-3 (Cell Signaling Technology, USA); p62 (Sigma, USA); $\beta$-actin (Proteintech, USA); horseradish peroxidase-conjugated antimouse IgG and anti-rabbit IgG (Cell Signaling Technology, USA). All primary and secondary antibodies used were diluted to $1: 1000$ and 1:2000 in 5\% skimmed milk, respectively. The protein bands of interest were visualized using an ECL kit (\#34079, Thermo Fischer Scientific Inc., USA) and GeneGnome5 (Syngene, UK). The staining intensity of the bands was determined and analyzed using Quantity One software (Bio-Rad).

\section{In situ hybridization}

Whole-mount in situ hybridization of chick embryos was performed according to a standard in situ hybridization protocol ${ }^{28}$. Digoxigenin-labeled probes were synthesized against VE-Cadherin ${ }^{21}$. The whole-mount stained embryos were photographed by a stereomicroscope (Olympus MVX10, Tokyo, Japan).

\section{RNA isolation and reverse transcription-PCR (RT-PCR)}

Total RNA was isolated from HH10 chick embryos using a Trizol kit (Invitrogen, USA) according to the manufacturer's instructions. First-strand complementary DNA (cDNA) was synthesized to a final volume of $25 \mu \mathrm{l}$ using SuperScript RIII first-strand (Invitrogen, USA). Following reverse transcription, PCR amplification of the cDNA was performed as described previously ${ }^{29,30}$. The sets of primers used for RT-PCR are described in the Supplementary Fig. 1. The PCR reactions were performed in a Bio-Rad S1000TM Thermal cycler (Bio-Rad, USA). The final reaction volume was $50 \mu \mathrm{l}$, comprising $1 \mu \mathrm{l}$ of first-strand cDNA, $25 \mu \mathrm{M}$ forward primer, $25 \mu \mathrm{M}$ reverse primer, $10 \mu \mathrm{l}$ PrimeSTARTM Buffer (Mg2+ plus), $4 \mu \mathrm{l}$ dNTP Mixture (TaKaRa, Japan), $0.5 \mu$ l PrimeSTARTM HS DNA Polymerase (2.5 U/ $\mu \mathrm{l}$ TaKaRa, Japan) and RNase-free water. cDNA was amplified for 30 cycles. One round of amplification was performed at $94{ }^{\circ} \mathrm{C}$ for $30 \mathrm{~s}$ and then $30 \mathrm{~s}$ at $58^{\circ} \mathrm{C}$ and $30 \mathrm{~s}$ at $72{ }^{\circ} \mathrm{C}$. The PCR products $(20 \mu \mathrm{l})$ were resolved using $1 \%$ agarose gels (Biowest, Spain) in $1 \times$ TAE buffer $(0.04 \mathrm{M}$ Trisacetate and $0.001 \mathrm{M}$ EDTA) and $10,000 \times$ GeneGreen Nucleic Acid Dye (TIANGEN, China) solution. The resolved products were visualized using a transilluminator (SYNGENE, UK), and photographs captured using a computer-assisted gel documentation system (SYNGENE). Each of these experiments was replicated at least three times.

\section{RNA isolation and quantitative PCR}

Total RNA was isolated from chick embryo (HH7 and HH10) HUVECs using a Trizol kit (Invitrogen, USA) according to the manufacturer's instructions. First-strand cDNA was synthesized to a final volume of $20 \mu \mathrm{l}$ using iScriptTM cDNA Synthesis Kit (Bio-Rad, USA). Following reverse transcription, PCR amplification of the CDNA was performed as described previously ${ }^{29,30}$. SYBR ${ }^{\circ}$ Green qPCR assays were then performed using a PrimeScriptTM RT reagent kit (Takara, Japan). All specific primers used are described in Supplementary Fig. 2. Reverse transcription and amplification reactions were performed in Bio-Rad S1000TM (Bio-Rad, USA) and ABI 7000 thermal cyclers, respectively. The housekeeping gene PPIA was run in parallel to confirm that equal amounts of RNA were used in each reaction. The ratio between the intensities of the fluorescently stained bands corresponding to genes and PPIA was calculated to quantify the level of the transcripts for those gene mRNAs.

\section{Quantitation of apoptotic cells}

Annexin V-FITC (BD Bioscience, USA) and PI double staining were used to identify and quantify apoptotic cells present in the HUVECs (control, $50 \mathrm{mM}$ glucose, $6 \mu \mathrm{M}$ Baicalin, $6 \mu \mathrm{M}$ Baicalin $+50 \mathrm{mM}$ glucose, $40 \mu \mathrm{g} / \mathrm{ml}$ Vitamin $\mathrm{C}+50 \mathrm{mM}$ glucose, $10 \mu \mathrm{M}$ chloroquine $+50 \mathrm{mM}$ glucose treated for $48 \mathrm{~h}$ ). Briefly, the cells were collected and resuspended in cold PBS at a density of $1 \times 10^{6}$ cells $/ \mathrm{ml}$ and then introduced into 200 $\mu \mathrm{l}$ of the Annexin V-binding buffer. The samples were then incubated with $2 \mu \mathrm{l}$ fluorescein isothiocyanate (FITC)-labeled Annexin V and $2 \mu \mathrm{l}$ PI at room temperature for $15 \mathrm{~min}$ and immediately analyzed by a FACS Calibur flow cytometer (BD, NJ, USA). The acquired data were evaluated using FCS-Express software version 3.0 (De Novo). 


\section{Measurement of oxidative stress}

Intracellular ROS was determined using a nonfluorescent dye DCF-DA $\left(2^{\prime}, 7^{\prime}\right.$-dichlorodihydrofluorescein diacetate) (Sigma, USA), which is oxidized by ROS to the fluorescent dye DCF (2',7'-dichlorofluorescin). The control and high glucose $(50 \mathrm{mM})$ and/or Baicalin $(6 \mu \mathrm{M})$-treated HUVECs were incubated in $10 \mu \mathrm{M}$ DCF-DA for $20 \mathrm{~min}$. Fluorescence was measured with a BD FACSAria (USA).

Superoxide dismutase (SOD), glutathione peroxidase (GSH-Px) and malonicdialdehyde (MDA) are expressed in the HH10 chick embryos. SOD, GSH-Px and MDA activities were elucidated using commercial kits (Nanjing, China). The results from control and the three treated groups were analyzed based on the differences of their respective fluorescein decay curves.

\section{Detection of intracellular chloride ion}

Intracellular chloride ion concentration was measured using $N$-[ethoxycar bonylmethyl]-6-methoxy-qu inolinium bromide (MQAE) (Beyotime, China), a fluorescent chloride ion indicator. Upon binding halide ions such as chloride, MQAE is quenched, resulting in a decrease in fluorescence without a shift in wavelength. HUVECs were incubated in Krebs-HEPES buffer (PanEra, China) containing $5 \mathrm{mM}$ MQAE at room temperature for $30 \mathrm{~min}$, and then washed three times with Krebs-HEPES buffer. MQAE-loaded cells (over $1 \times 10^{5}$ cells) were then mounted on a glass coverslip for $20 \mathrm{~min}$ at room temperature.

\section{Data analysis}

The BVD in the CAM, YSM or blood island density was analyzed using the Image Pro-Plus 5.0 software $^{20}$. Statistical analyses for all the experimental data generated were performed using a SPSS 13.0 statistical package program for Windows. The data were presented as mean \pm SD. Statistical significance were determined using paired Test, independent samples $T$-test or one-way analysis of variance. $P<0.05$ was considered to be significant.

\section{Results}

\section{Screening the optimal concentration of Baicalin for administration to early chick embryos}

To determine whether or not there is a cytotoxic effect on developing embryos following the application of Baicalin $^{31}$, HHO chick embryos were first incubated with 3, 6, 12 and $24 \mu \mathrm{M}$ Baicalin for 26,39 and $48 \mathrm{~h}$ in EC culture (Fig. 1a), respectively. The developing chick embryos treated with sample saline (control) or various concentrations $(3,6,12$ and $24 \mu \mathrm{M})$ of Baicalin were photographed at incubation times of $0,26,39$ and $48 \mathrm{~h}$ (Fig. 1b). Using embryo length and somite pair number as the embryonic developmental indices, 3 and $6 \mu \mathrm{M}$ Baicalin showed no effect, but exposure to 12 and $24 \mu \mathrm{M}$ Baicalin significantly reduced length development $(p<0.05, p<0.01, p<0.001$; $n>6$ embryos in each group) and somite pair numbers in comparison to control embryos $(p<0.01, p<0.001 ; n=$ 10; Fig. 1b1-b4). Meanwhile, it was observed that $3 \mu \mathrm{M}$ and $6 \mu \mathrm{M}$ Baicalin had no obvious effect on embryonic malformation, but higher exposures of 12 and $24 \mu \mathrm{M}$ had a significant effect (severe malformation: $33 \%$ in $12 \mu \mathrm{M}$ and $50 \% 24 \mu \mathrm{M}, n>6$ embryos in each group; Fig. 1c-c1). Therefore, concentrations of 3 and $6 \mu \mathrm{M}$ were used in subsequent experiments.

\section{Baicalin administration successfully reversed hyperglycemia-inhibited development of early chick embryos}

Hyperglycemia has been shown to negatively affect embryo development ${ }^{32}$. Here, through comparing the development of gastrula chick embryos exposed to $50 \mathrm{mM}$ glucose (high glucose (HG)) and/or 3 or $6 \mu \mathrm{M}$ Baicalin for 26, 39 and $48 \mathrm{~h}$ (Fig. 2a), it was discovered that HG dramatically inhibited the length and somite pair numbers of chick embryos ( $p<0.001 ; n>6$ embryos in each group). However, the addition of $6 \mu \mathrm{M}$ Baicalin could significantly reverse the inhibitive effect on embryo development in 48 h $(p<0.001$ compared with the HG group; $n>6$ embryos in each group; Fig. 2a1-b). Simultaneously, the numbers of malformed embryos were also reduced to some degree after addition of $6 \mu \mathrm{M}$ Baicalin (severe malformation: 0\% in control, $50 \%$ in $\mathrm{HG}, 50 \%$ in $\mathrm{HG}+3 \mu \mathrm{M}$ Baicalin and $20 \%$ in HG+6 $\mu$ M Baicalin, $n>6$ embryos in each group, Fig. 2b). This indicates that Baicalin administration exerts a positive effect on hyperglycemia-inhibited embryo development.

\section{Baicalin administration saved hyperglycemia-induced malformation of early chick embryonic cardiovascular system}

Previous studies have shown that hyperglycemia could enhance the risk of heart tube malformation during embryogenesis $^{22}$. MF-20 immunofluorescent staining showed that hyperglycemia induced a high incidence of cardiac bifida (32\%, Fig. 3c-c2,e) which was clearly suppressed by addition of $6 \mu \mathrm{M}$ Baicalin (16\%, Fig. 3d-d2,e). Normal C-loop heart tubes could only be observed in the control and $6 \mu \mathrm{M}$ Baicalin groups $(100 \%, n=25$ in each group, Fig. 3a-a2,b-b2,e). The RT-PCR data showed that the expressions of heart tube formation-related genes, including $\mathrm{VMHC}, \mathrm{N}$-Cadherin, Wnt3a and $\mathrm{BMP} 2$, were down-regulated in the presence of $\mathrm{HG}(p<0.01, p<$ 0.001), but VMHC, N-Cadherin, Wnt3a and BMP2 recovered to an extent following addition of $6 \mu \mathrm{M}$ Baicalin ( $p<0.01, p<0.001$ compared to the HG group; Fig. 3f- $\mathrm{f} 1$ ). A similar tendency was observed in the expression of GATA4 as indicated by western blot data $(p<0.05$ compared with the control group, $p<0.01$ compared with the HG group, Fig. 3g). Most of these genes were not 


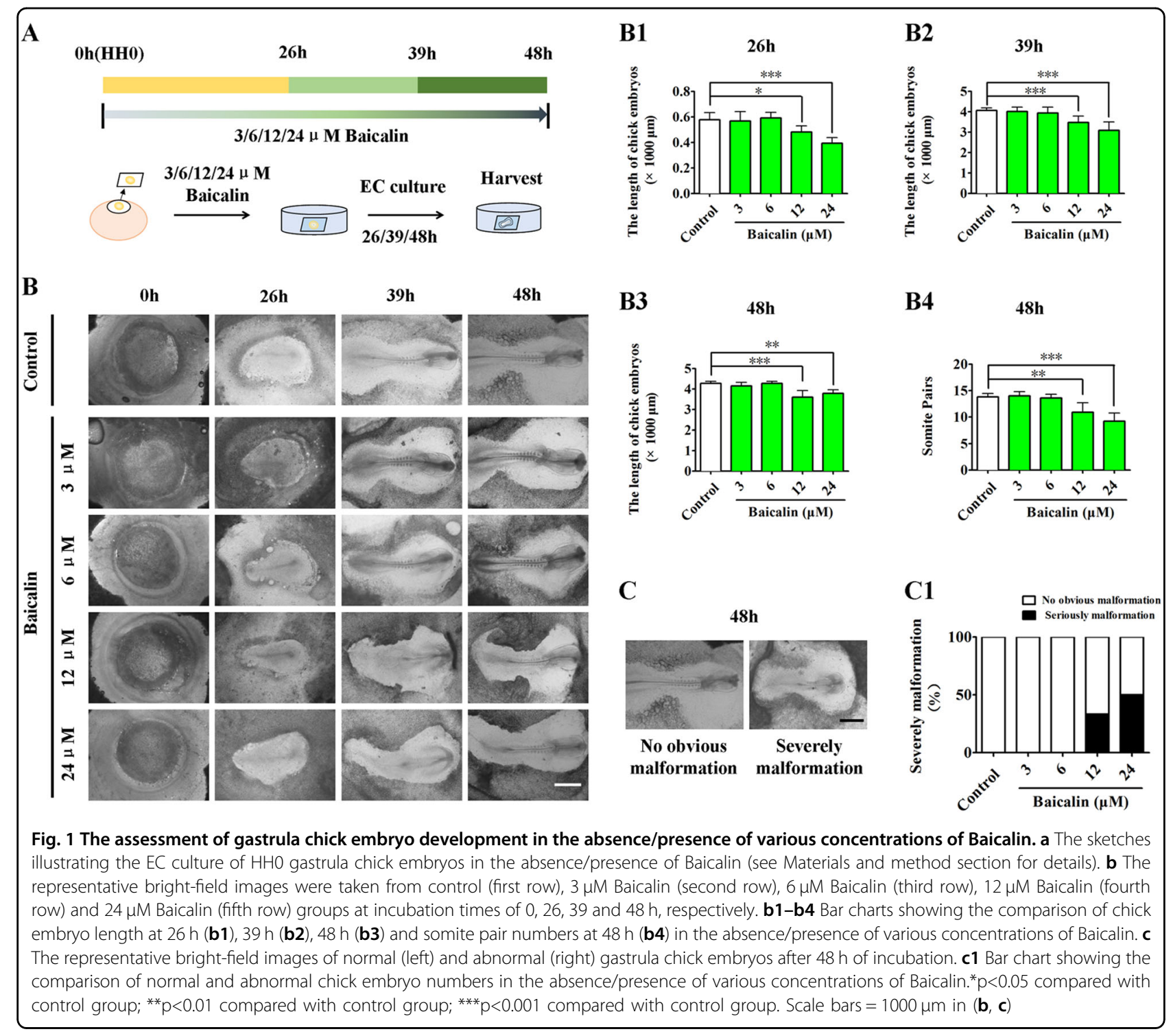

changed significantly in the Baicalin group compared with the control group $(p>0.05)$.

Vasculogenesis starts from blood islands expressed in VE-Cadherin, the blood island marker. VE-Cadherin in situ hybridization in whole-mount HH8 chick embryos (Fig. 4a) showed that blood island formation was not affected by $6 \mu \mathrm{M}$ Baicalin $(p>0.05$, Fig. $4 \mathrm{~b}-\mathrm{c}, \mathrm{b} 1-\mathrm{c} 1$, b2-c2,f), was suppressed by $50 \mathrm{mM}$ glucose $(p<0.001)$, but the reduced blood islands increased again when Baicalin and glucose were applied together ( $p<0.01$ compared with HG group, $n=8$ in each group, Fig. 4d-e,d1e1,d2-e2,f). This suggests that Baicalin administration could rescue the hyperglycemia-inhibited vasculogenesis to some extent.

Furthermore, whether or not Baicalin administration could rescue HG-inhibited angiogenesis ${ }^{23}$ was studied using the chick CAM model, illustrated in Fig. S2A. Chick embryo weight on the harvest day (day 9) was increased slightly when exposed to $6 \mu \mathrm{M}$ Baicalin $(p>0.05)$, and reduced when exposed to $50 \mathrm{mM}$ glucose $(p<0.05)$, but this reduction was reversed after exposure to $6 \mu \mathrm{M}$ Baicalin $(p<0.01$ compared with HG group, $n>10$ embryos in each group; Fig. S2B). Compared to the angiogenesis on CAM in control group ( $p<0.05$, Fig. S2C), it was clear that $6 \mu \mathrm{M}$ Baicalin alone did not change the blood vessel density ( $p>0.05$, Fig. S2D), and that $50 \mathrm{mM}$ glucose dramatically inhibited angiogenesis $(p<0.05$, Fig. S2E). The combination of $6 \mu \mathrm{M}$ Baicalin and $50 \mathrm{mM}$ glucose significantly reversed the reduction of blood vessel density induced by HG ( $p<0.05$ compared with HG group, $n>8$ in each group; Fig. S2F). This is quantitatively analyzed in Fig. S2G. 


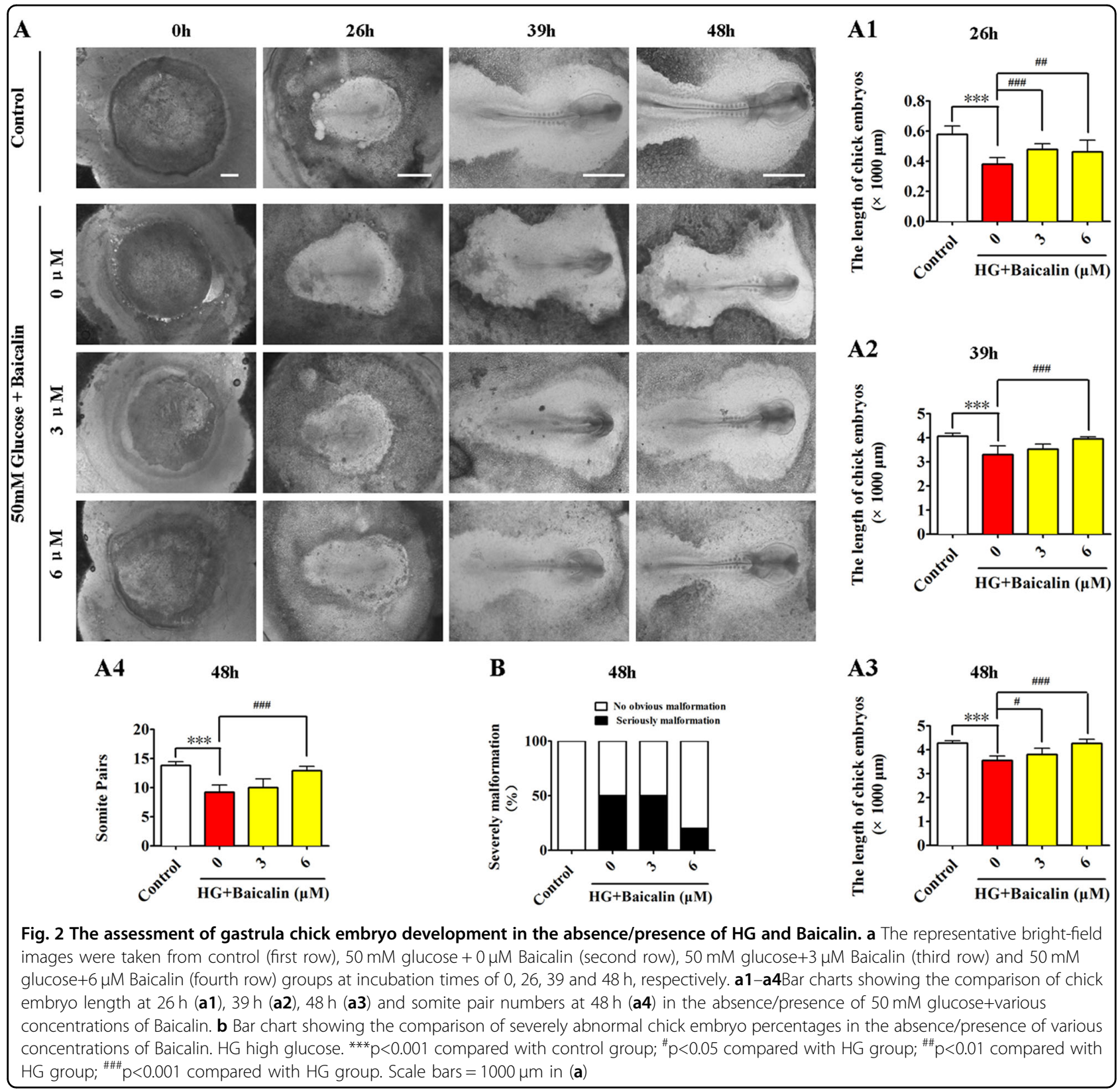

In addition, the RT-PCR data showed that expressions of vasculogenesis-related genes VEGF-R2 were downregulated in the presence of HG $(p<0.01)$, but there was a partial recovery after addition of $6 \mu \mathrm{M}$ Baicalin $(p<0.05$ compared with HG group, Fig. 3g).

\section{Baicalin administration rescued hyperglycemia-induced cell proliferative reduction and apoptosis increase}

Western blot data showed that the expression of apoptosis-related gene C-caspase- 3 increased in the presence of HG $(p<0.001)$, but was reversed to some extent by addition of $6 \mu \mathrm{M}$ Baicalin ( $p<0.01$ compared with HG group, Fig. 5a) in early chick embryo. Furthermore,
HUVECs were incubated with $6 \mu \mathrm{M}$ Baicalin $/ 50 \mathrm{mM}$ glucose or in combination for 12, 24, 36 and $48 \mathrm{~h}$. The cell counting kit-8 (CCK8) was then used to detect cell proliferation and viability (Fig. 5b). The results showed that the HUVEC proliferation and viability dropped in the presence of $\mathrm{HG}$ at 12, 24, 36 and $48 \mathrm{~h}$ of incubations $(p<$ $0.001)$, but all of these reductions increased again when 6 $\mu \mathrm{M}$ Baicalin and $50 \mathrm{mM}$ glucose $(p<0.001$ was added together compared with HG group, $n>8$ in each group; Fig. 5b). The data from flow cytometry showed that 50 $\mathrm{mM}$ glucose could significantly enhance HUVEC apoptosis $(p<0.001)$, and this increased apoptosis induced by HG was dramatically suppressed by the addition of $6 \mu \mathrm{M}$ 


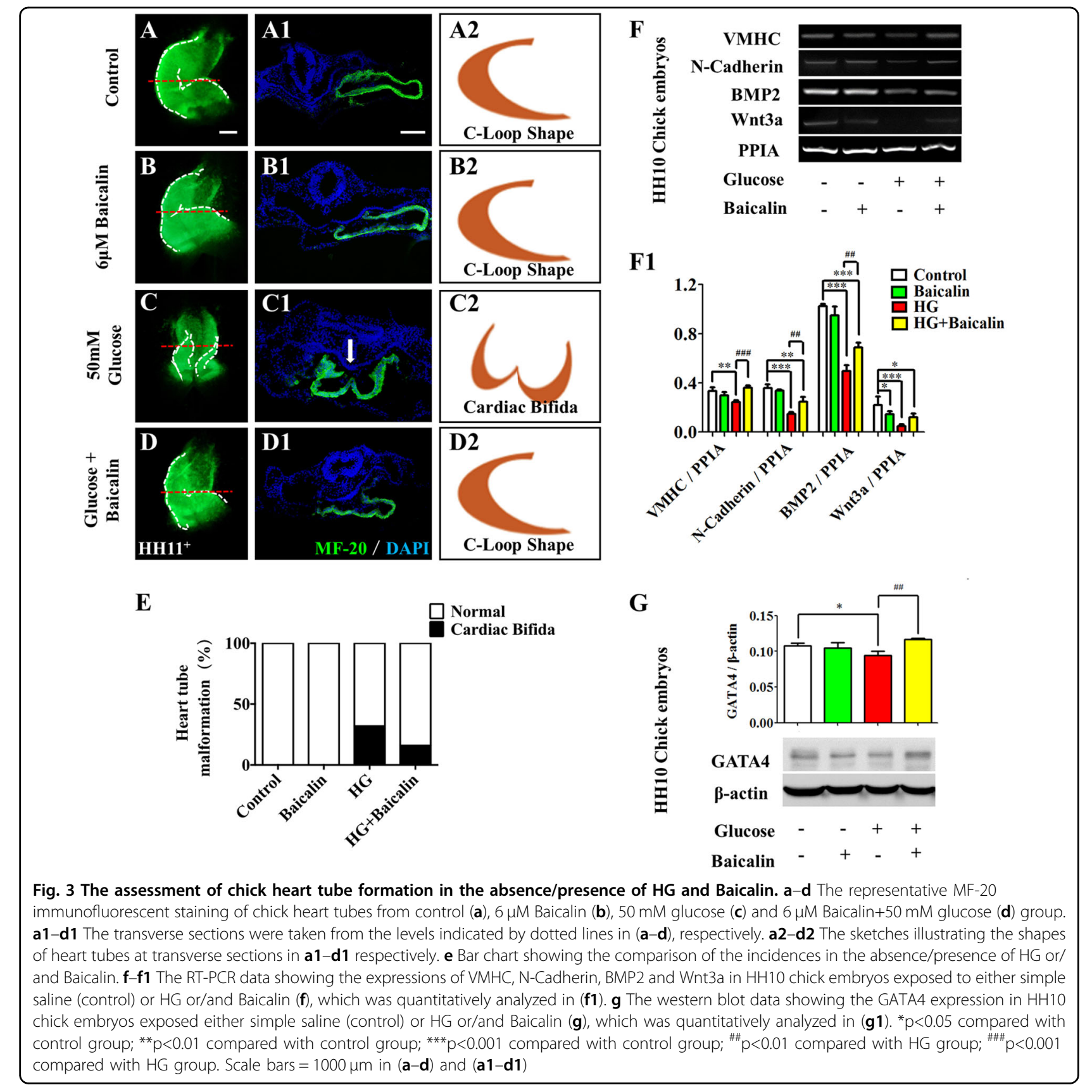

Baicalin $(p<0.05$ compared with HG group, $n=3$ in each group, Fig. 5c-c1). Furthermore, using PI fluorescent staining, it could be demonstrated that $50 \mathrm{mM}$ glucose administration greatly increased the $\mathrm{PI}^{+}$HUVEC numbers $(p<0.001)$, whereas HG-induced elevation of $\mathrm{PI}^{+}$ HUVEC numbers basically returned to normality $(p<$ 0.001 compared with HG group, $n=3$ in each group, Fig. 5d-h,d1-g1). Meanwhile, the HUVEC shape outlined by $\mathrm{F}$-actin staining was observed to shrink in the presence of $50 \mathrm{mM}$ glucose $(p<0.001, n>18$ in each group, Fig. 5d-h,d2-g2), and then it generally kept in size when additional $6 \mu \mathrm{M}$ Baicalin was added $(p<0.001$ compared to the HG group, $n>18$ in each group, Fig. $5 \mathrm{i}$ ).

\section{Baicalin administration stabilized the hyperglycemia- induced oxidative stresses' secondary effect on angiogenesis}

The obviously excessive ROS production was discovered in the presence of $50 \mathrm{mM}$ glucose in HUVECs in vitro culture $(p<0.001, n=5$ in each group), but the addition of $6 \mu \mathrm{M}$ Baicalin returned ROS levels back to normal $(p<0.01$ compared with HG group, Fig. 6a). 


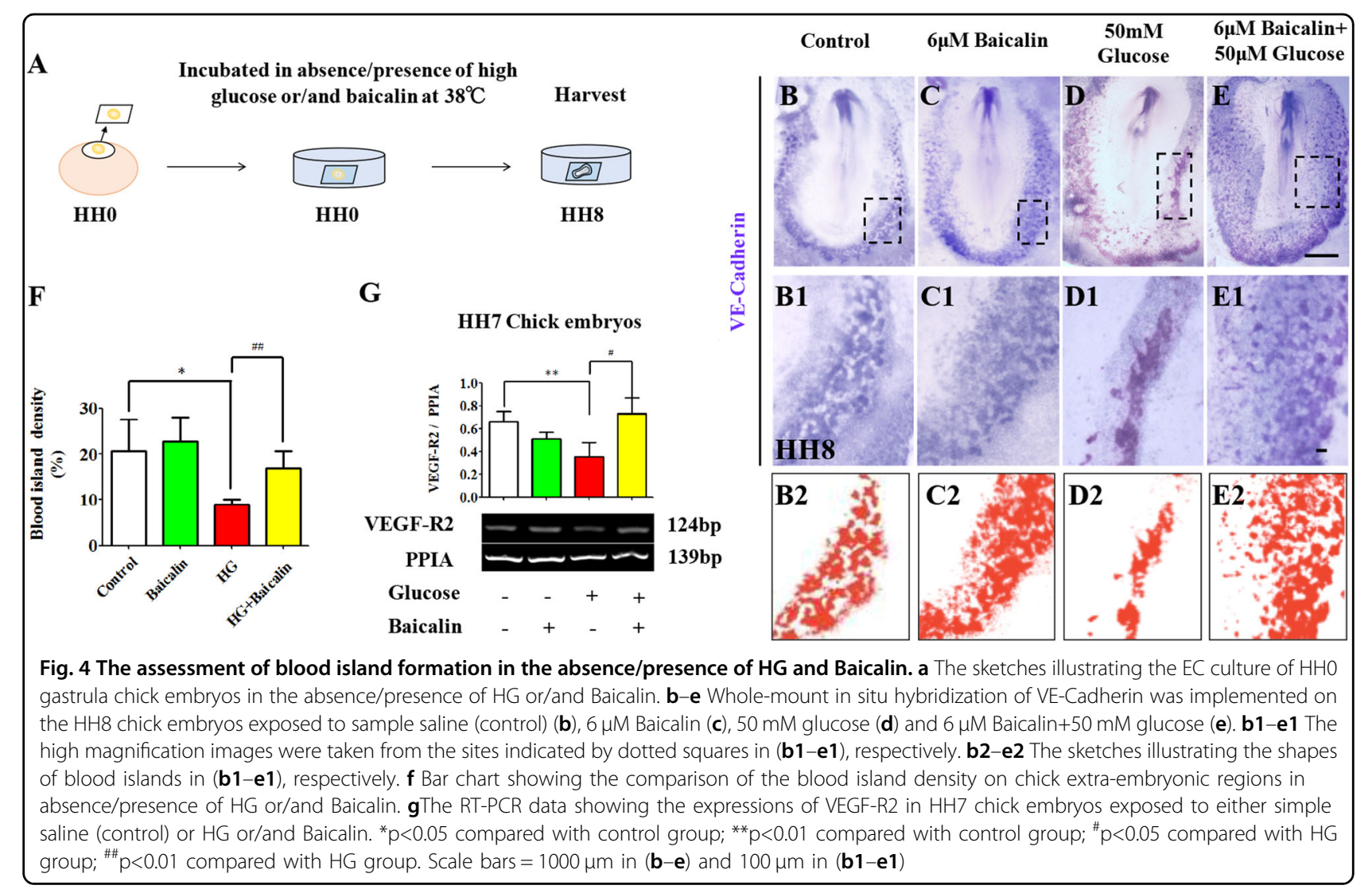

Staining with DHE, the cell-permeable fluorescent redox indicator of superoxide, demonstrated that $50 \mathrm{mM}$ glucose administration in HUVECs could significantly increase the level of superoxide, but cotreatment with 6 $\mu \mathrm{M}$ Baicalin and $50 \mathrm{mM}$ glucose could restore the level of superoxide $(p<0.05$, Fig. 6b-b1). Additionally, the activities of SOD, MDA and GSH-Px were measured in HH10 chick embryos exposed to either Baicalin, glucose or in combination. The results demonstrated that MDA activity was distinctly elevated in the presence of $50 \mathrm{mM}$ glucose $(p<0.001$, Fig. $6 \mathrm{c})$, but dropped sharply after addition of $6 \mu$ M Baicalin $(p<0.001$ compared with HG group, $n=3$ in each group). There were no significant alterations to SOD activities when the embryos were exposed to HG ( $p$ $>0.05, n=8$, Fig. $6 \mathrm{~d}$ ), but dropped significantly when treated with HG+Baicalin $(p<0.001, n=8$, Fig. $6 \mathrm{~d})$. GSH-Px activity was elevated in the presence of $50 \mathrm{mM}$ glucose $(p<0.01$ Fig. 6 e), but the enzyme activities were decreased following addition of $6 \mu \mathrm{M}$ Baicalin in the presence of $50 \mathrm{mM}$ glucose $(p<0.001$ compared with HG group, $n>6$ in each group, Fig. 6e). MQAE staining for intracellular chloride ion concentration showed that there more fluorescent spots in the presence of HG, but were partially recovered after the addition of $6 \mu \mathrm{M}$ Baicalin in HUVECs (Fig. S3, yellow arrows). Quantitative PCR data showed that the expressions of GABAA were up- regulated in the presence of HG $(p<0.01)$, but were partially recovered after addition of $6 \mu \mathrm{M}$ Baicalin in chick embryos $(p<0.001$ compared with HG group, $n=3$, Fig. 6f).

Next, the environment of excessive ROS level was induced by the application of AAPH, a ROS generator, in chick YSM. AAPH $(3.75 \mathrm{mg} / \mathrm{ml})$ treatment resulted in the deaths of $13.40 \%$ of chick embryos as shown in Fig. $6 \mathrm{~g}$-g1, but only $6.67 \%$ chick embryo deaths were found on addition of $6 \mu \mathrm{M}$ Baicalin after AAPH exposure $(n=30$ embryos in each group). AAPH administration could significantly inhibit angiogenesis in YSM $(p<0.05)$, whereas a lesser effect was obtained following combined application of AAPH and Baicalin $(p<0.001, n>7$ in each group, Fig. 6h-h1), suggesting that oxidative stress could be one of the mechanisms underlying this protective effect on hyperglycemia-inhibited angiogenesis.

\section{Baicalin administration redressed the hyperglycemia- induced unbalanced secondary effect of autophagy on heart tube formation}

The western blot data showed that the ratio of LC3II/ LC3I, Beclin1 and p62 were highly expressed in the presence of $50 \mathrm{mM}$ glucose $(p<0.05, p<0.01)$, and that the HG-enhanced expressions were suppressed in HH10 chick embryos by the administration of $6 \mu \mathrm{M}$ Baicalin ( $p$ 


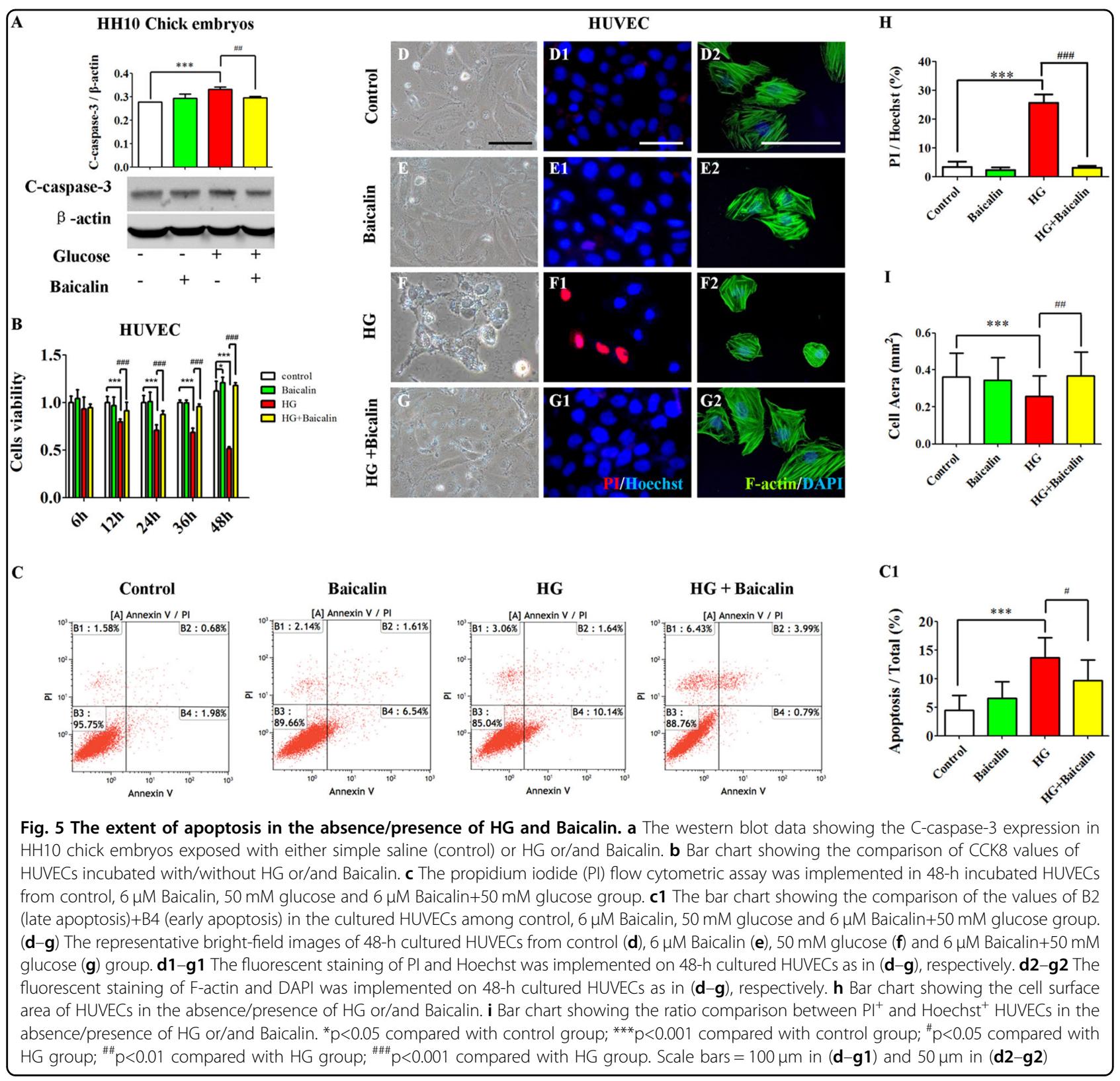

$<0.01, n=3$, Fig. 7a-a1). When Rapa was used to induce autophagy in early gastrula chick embryos, $28 \%$ cardiac bifida mortality of chick embryos was found $(n=25$ in each group, Fig. $7 \mathrm{~b}-\mathrm{e}$ ) but this was reduced to $12 \%$ when $6 \mu \mathrm{M}$ Baicalin was added.

To distinguish whether or not there are different roles of oxidative stress and autophagy in hyperglycemiainduced cardiovascular malformation, $\mathrm{PI}^{+}$cell numbers were determined and PI flow cytometric assays conducted in HUVECs in the presence of HG alone and together with either Baicalin, Chloroquine (CQ) or Vitamin C (VC, Fig. 8). The results indicate that HG significantly elevated cell $\mathrm{PI}^{+}$cell numbers compared with control group $(p<$ $0.001, n=3$ in each group. Fig. 8a-b1,f); the addition of either $6 \mu \mathrm{M}$ Baicalin or VC suppressed this elevation $(p<$ 0.001 compared with HG group, $n=3$. Fig. $8 \mathrm{c}-\mathrm{c} 1$,e-e1,f). However, the addition of CQ did not reduce the HGinduced increase of cell $\mathrm{PI}^{+}$cell numbers but were even increased further $(p>0.05$ compared with HG group, Fig. $8 \mathrm{~d}$-d1,f). The flow cytometry data showed that $50 \mathrm{mM}$ glucose could significantly enhance HUVEC apoptosis ( $p$ $<0.01, n=3$ ), and the increased apoptosis induced by HG was dramatically suppressed by the addition of $6 \mu \mathrm{M}$ Baicalin or $\mathrm{VC}(p<0.01, p<0.05$ compared with HG 


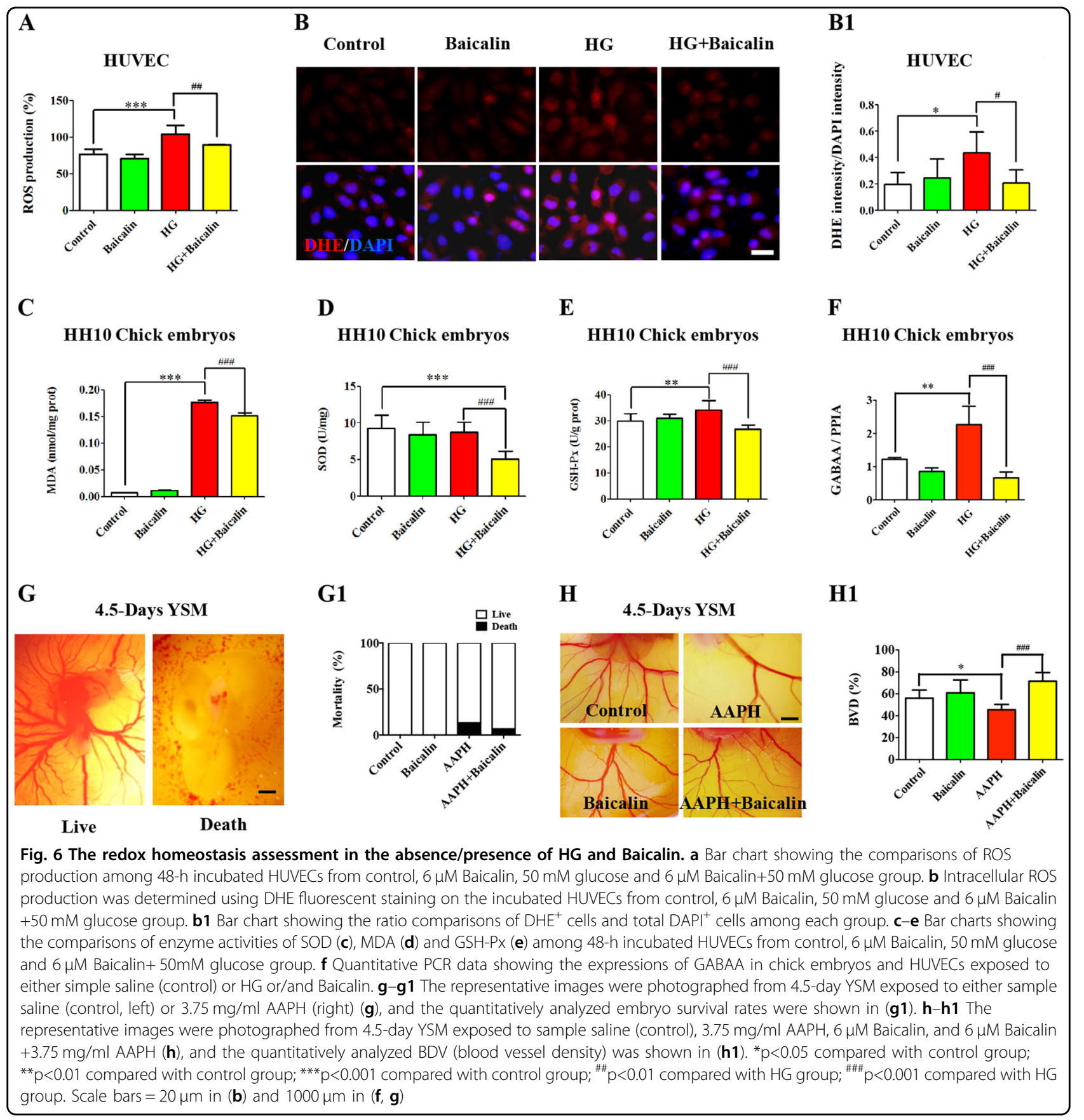

group, $n=3$, Fig. $8 \mathrm{~h}-\mathrm{i}, \mathrm{k}-\mathrm{l})$. However, addition of CQ failed to reduce the HG-induced apoptosis $(p<0.01$ compared with control group, Fig. 8j,l) and $\mathrm{PI}^{+}$cell numbers were again increased $(p>0.05$ compared with HG group, Fig. $8 \mathrm{~h}, \mathrm{j}, \mathrm{l})$.

\section{Baicalin administration in pregnant mice could partially suppress STZ-induced hyperglycemia}

In order to examine whether the protective effect of Baicalin is a consequence of modulating maternal blood levels, pregnant STZ-induced diabetes mellitus mice were treated as shown in Fig. 9a. The blood glucose level was found to be significantly increased after 3 days of STZ injection, compared with the controls (second week: $p<0.001, n=25$, Fig. 9b). Diabetic mice were then randomly divided into two groups and one group was treated with Baicalin for 10 days. Compared to the diabetic controls, the blood glucose levels in the the group receiving Baicalin by intra-gastric administration were significantly lower after 3 weeks $(p<0.01$ compared with 
A

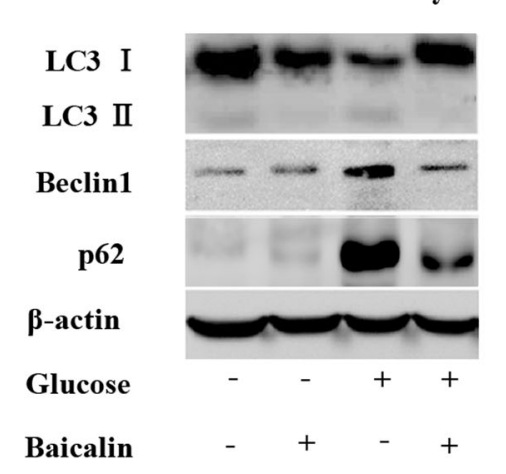

\section{A1}

$16 \mathrm{kDa}$

14kDa

$60 \mathrm{kDa}$

$62 \mathrm{kDa}$

42kDa

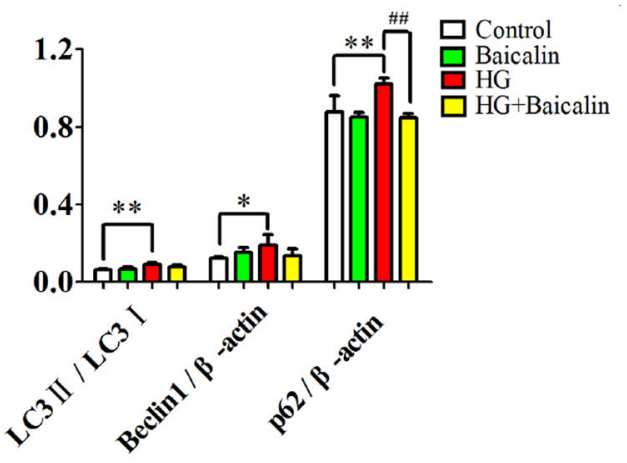

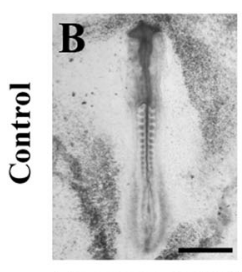
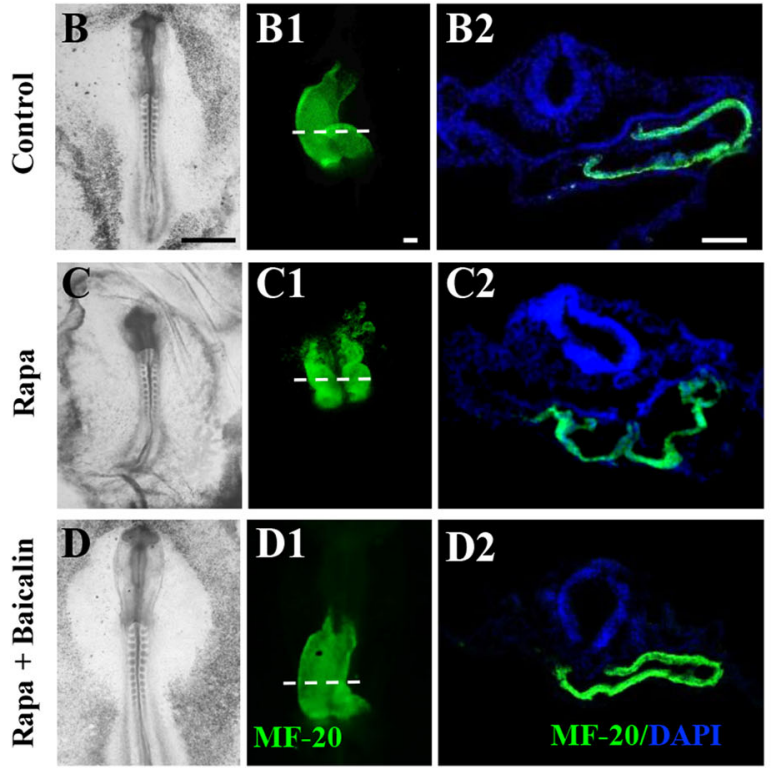

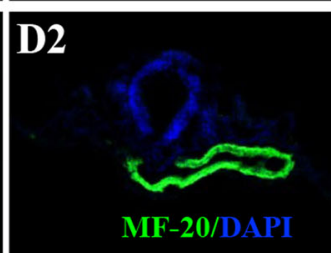

$\mathbf{E}$

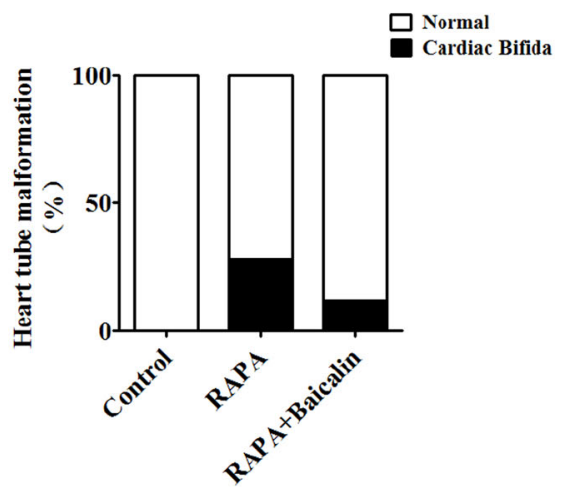

Fig. 7 The assessment of autophagy in gastrula chick embryos and the incubated HUVECs in the absence/presence of Baicalin. a-a1 Western blot data showing the expressions of LC3I, LC3II, p62 and Beclin1 in the opaca area of HH10 chick embryos from control, $6 \mu \mathrm{M}$ Baicalin, 50 $\mathrm{mM}$ glucose and $6 \mu \mathrm{M}$ Baicalin+50 mM glucose group (a), which was quantitatively analyzed in (a1). b-d The representative bright-field images of HH10 chick embryos exposed to sample saline (control) (b), Rapa (c) or Rapa+Baicalin (d). b1-d1 The MF-20 immunofluorescent staining was implemented in HH10 chick embryos exposed to sample saline (control) (b1), Rapa (c1) or Rapa+Baicalin (d1). b2-d2 The transverse sections at the levels indicated by dotted lines in (b1-d1), respectively. e Bar chart showing the comparison of cardiac bifida incidences in HH10 chick embryos exposed to sample saline (control), Rapa or Rapa+Baicalin. ${ }^{*} p<0.05$ compared with control group; ${ }^{* *} p<0.01$ compared with control group; ${ }^{\# \#} p<0.01$ compared with HG group. Scale bars $=100 \mu \mathrm{m}$ in (b-d), $10 \mu \mathrm{m}$ in (b1-d1) and $100 \mu \mathrm{m}$ in $(\mathbf{g})$

the diabetes mellitus group, $n=11,12$, Fig. 9b). However, there was no significant difference at the 4-5-week stage. The histologies of kidneys and livers were then examined on the transverse sections. In comparison to the kidney control, endothelium damage was observed (Fig. 9c2), together with glomerular sclerosis (Fig. 9c5), hyperplasic glomeruli mesangial cells (Fig. 9c5), thickening of glomerular basement membrane (Fig. 9c8) as shown by stars, but such kidney structure damage was much less apparent in the mice receiving Baicalin (Fig. 9c3,c6,c9). Increases of glomerular sclerosis (diabetes mellitus group compared with control group: $p<0.001$, Baicalin administration group compared with diabetes mellitus group: $p<0.01$, Fig. 9d) and mesangial area (diabetes mellitus group compared with control group: $p<0.001$, Baicalin administration group compared with diabetes mellitus group: $p<0.01$, Fig. 9e) showed that diabetes was suppressed by Baicalin. Likewise, the degeneration and necrosis of hepatocytes in the transverse section of diabetes mellitus mouse liver compared to control was dramatically improved after Baicalin administration (Fig. 9f). These protective effects on kidney and liver might contribute to lowering elevated blood glucose levels in diabetic mice. 


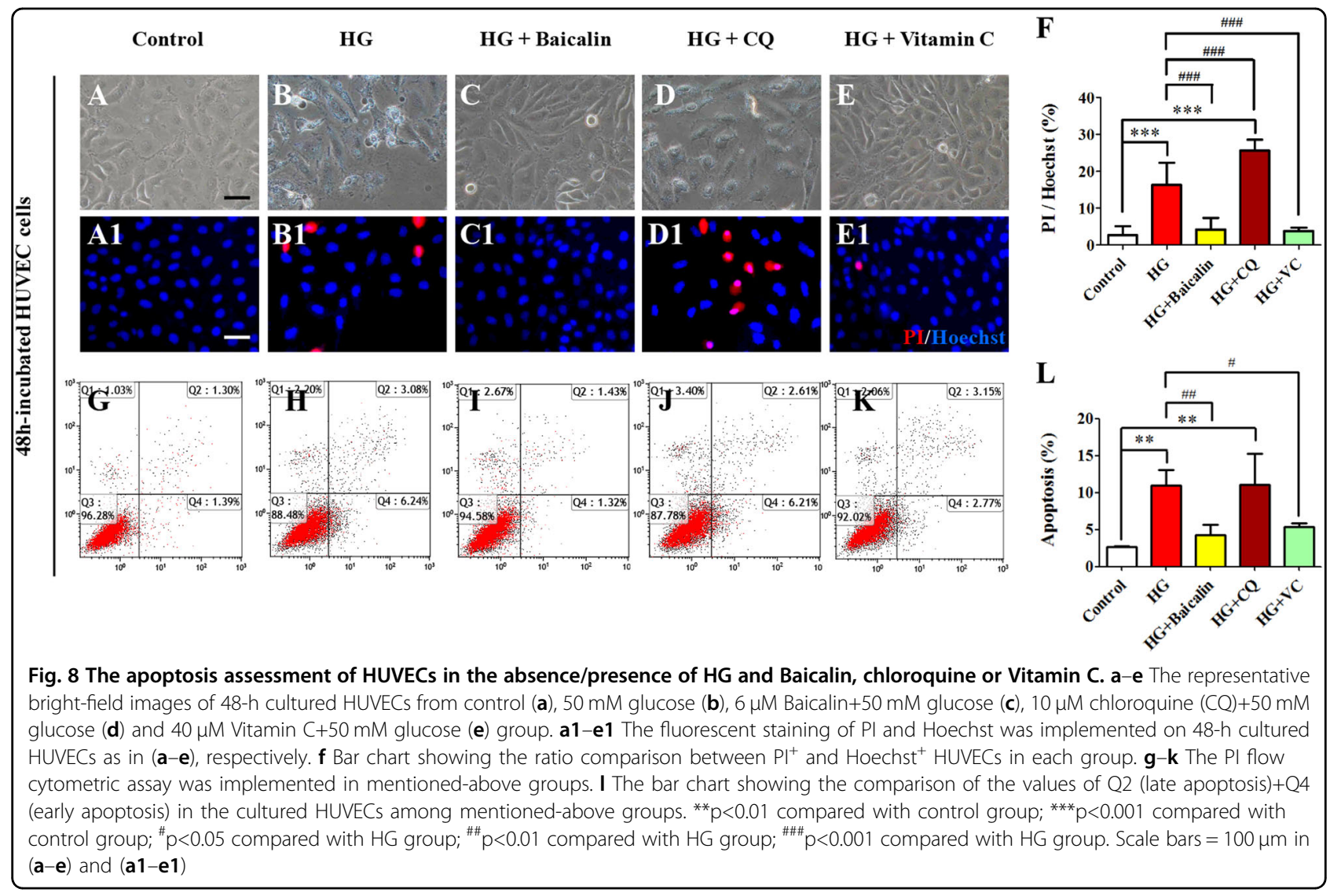

\section{Discussion}

Baicalin is present as a glycoside in Scutellaria baicalensis Georgi, and is metabolized to its aglycone, Baicalein, by the action of intestinal $\beta$-glucuronidase in the intestine $^{33}$. Significantly, Baicalin can be reformed into Baicalein, which is its previous metabolism, in many tissues. Both Baicalin and Baicalein can be detected in blood after absorption in the intestine ${ }^{34}$. The roots of Scutellaria baicalensis Georgi have been extensively used as traditional medicines in many East Asian countries to reduce inflammation ${ }^{35}$. Earlier studies have shown Baicalin and Baicalein to possess wide-ranging biological and pharmacological functions, including anti-inflammation, anticancer and anti-pruritic effects ${ }^{36-38}$. Most experiments have been carried out in vitro only, which simplifies the scientific protocols but makes extrapolation to the in vivo situation difficult, if not impossible. Therefore, it is crucial to conduct well-designed in vivo experiments to investigate these compounds' pharmacological and toxicological effects. As one of the first systems to develop in early pregnancy, the cardiovascular system is undoubtedly vulnerable to diabetes mellitus-induced hyperglycemia exposure. Dysplasia of the cardiovascular system during cardiogenesis and angiogenesis leads to constantly impaired fetal development or death. Therefore, in this study, the focus has been on whether Baicalin administration could improve hyperglycemia-induced cardiovascular dysplasia.

Most of the experiments in this study have been carried out using chick embryos. The advantage of this model is that chick embryos can grow outside of uterus. They can thus be easily manipulated, and can be especially useful for the study of effects on early embryo development in EC culture or angiogenesis using YSM and CAM. The chick embryo model allows direct monitoring of the development of the embryos at any stage following the experimental manipulation in either EC culture or windowed eggshell ${ }^{39}$. Song et al. ${ }^{40}$ reported the potential embryotoxicity of Baicalin when they evaluated the safety of Shuanghuanglian injection powder, in which Baicalin is the main component, through placental barriers of rats. The placental permeability of Baicalin undoubtedly enables it to adversely affect embryonal development after early embryo exposure.

For this reason, embryotoxic experiments were initially conducted. Using the EC culture approach, an optimal concentration of Baicalin was selected for administration in subsequent experiments that did not influence chick embryo development (Fig. 1). At the same time, hyperglycemia-inhibited embryo development was 


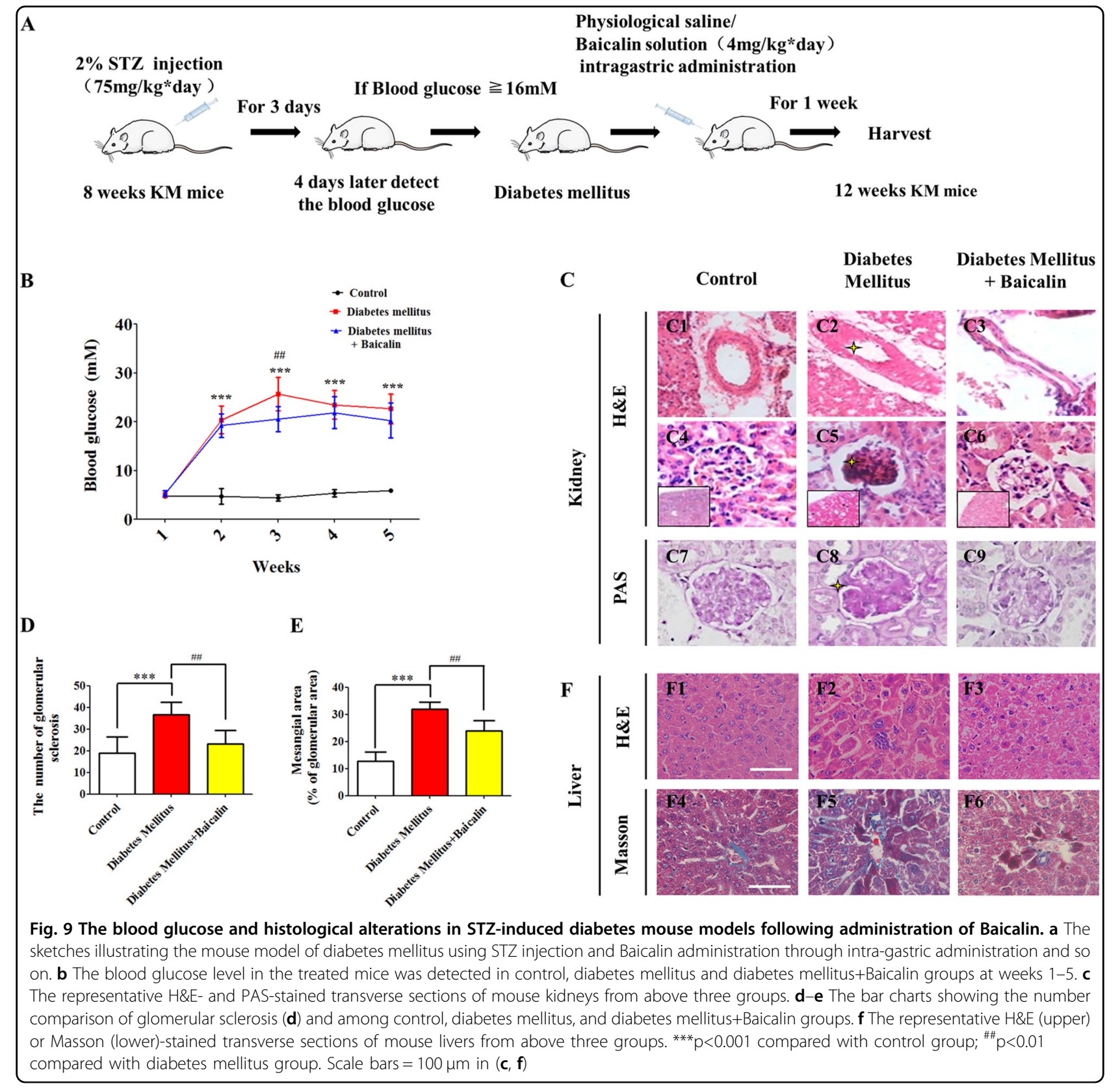

significantly attenuated (Fig. 2). In the process of heart tube formation, Baicalin administration could distinctly reduce the risk of hyperglycemia-induced cardiac bifida by reversing the expressions of the crucial genes related to heart tube development (Fig. 3). In evaluating the effect of Baicalin on vasculature development, the blood island formation model (vasculogenesis) in area opaca and the CAM model (angiogenesis), which include early stage of blood islands/primary vessel plexus (Fig. 4), were employed along with the later stage of angiogenesis (Fig. S2). Obviously, the inhibitive effect of hyperglycemia on blood island formation was greatly reduced in the presence of Baicalin (Fig. 4), while hyperglycemia-induced angiodysplasia on CAM was also minimized by Baicalin administration (Fig. S2). Taken together, these data suggest that Baicalin administration has a positive impact on hyperglycemia-induced cardiovascular dysplasia during early embryo development.

Hyperglycemic conditions can increase both apoptotic cell death and intracellular ROS generation ${ }^{41,42}$. Therefore, an investigation of whether apoptosis is a key link for Baicalin against hyperglycemia-induced cardiovascular 


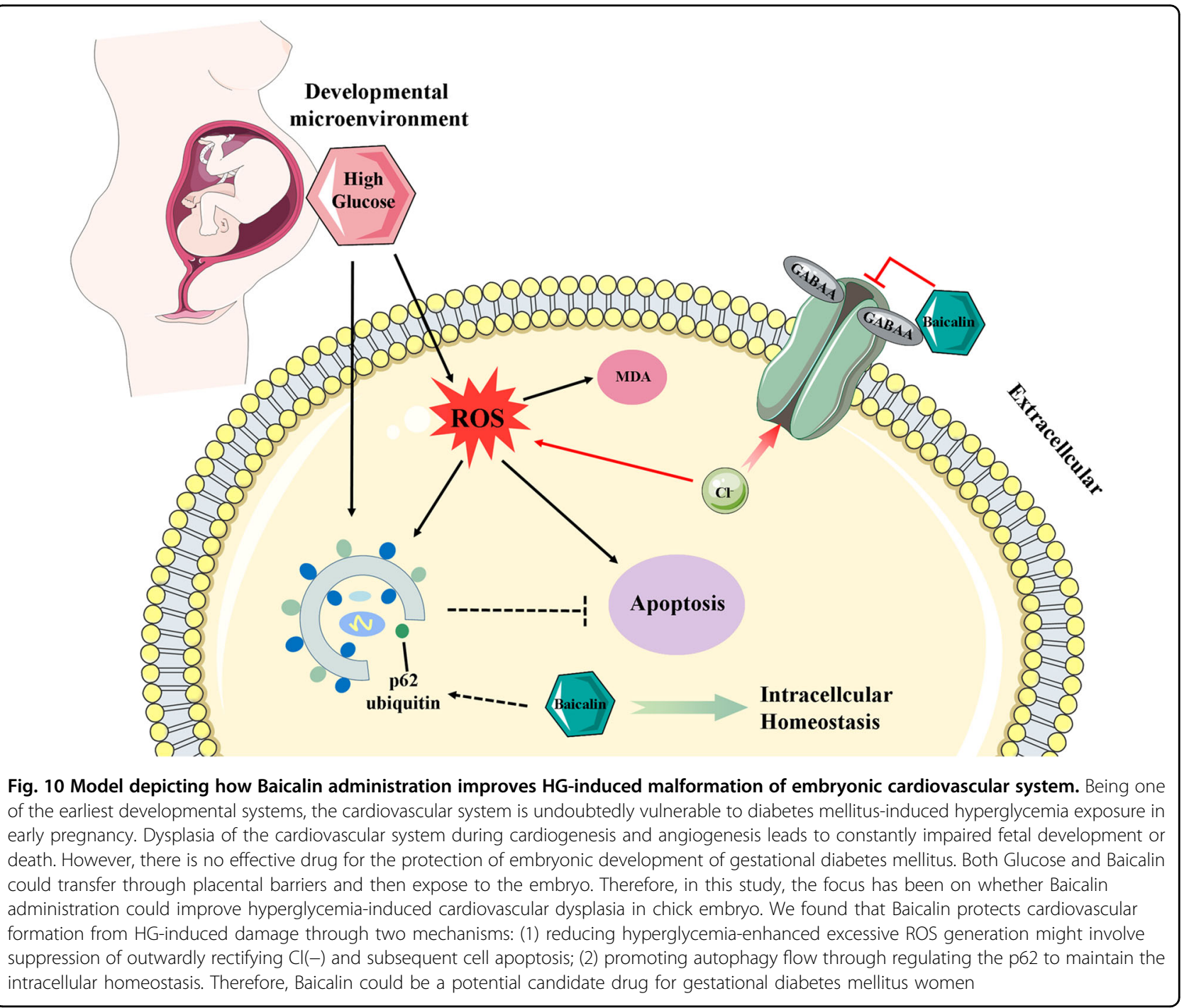

malformation was carried out. Cell apoptosis induced by hyperglycemic conditions was dramatically reversed by Baicalin administration (Fig. 5). SOD, GSH-Px and MDA were all decreased after addition of Baicalin to HG-treated chick embryos (Fig. 6c-e). This suggested that Baicalin might also play an antioxidant role in chick embryos, but not through activating SOD and GSH-Px. Cell size was reduced after HG exposure (Fig. 5i). The loss of cell volume or cell shrinkage is a known morphological hallmark of apoptosis ${ }^{43,44}$. Activation of the volume-sensitive outwardly rectifying $\mathrm{Cl}(-)$ channel is involved in this process $^{45}$. Detection of intracellular chloride ion showed a reduction of $\mathrm{Cl}(-)$ in the presence of $\mathrm{HG}$ and that can be reversed by the addition of Baicalin (Fig. S3). GABAA, an important $\mathrm{Cl}(-)$ channel, is sensitive to $\mathrm{ROS}$ and recent studies have demonstrated that Baicalin is acting through GABAA $^{5}$. The changes of GABAA shown here (Fig. 6f) suggested that the antioxidant function of Baicalin is caused by suppression of outwardly rectifying $\mathrm{Cl}(-)$ in the HG microenvironment. Meanwhile, both the in vivo and in vitro experiments imply that the hyperglycemiainduced angiodysplasia could be partially rectified through suppressing excessive ROS production induced by HG (Fig. 6g,h). These observations further confirm the antioxidant bioactivity of Baicalin ${ }^{8,46}$.

Autophagy has been shown to be involved in high glucose-induced heart tube malformation ${ }^{22}$. As in earlier reports ${ }^{47,48}$ high glucose was observed to increase the expression of p62, suggesting that autophagy flux is inhibited. However, after Baicalin was added, the expression of the hyperglycemia-enhanced autophagy gene decreased and cardiac bifida induced by Rapainduced excessive autophagy disappeared (Fig. 7). These results suggest that Baicalin might increase the ubiquitin of p62 and hence accelerate autophagy flux. These processes could facilitate the cleanup of damaged 
organelles. In order to examine this further and determine correlations between oxidative stress and autophagy, the effect of Baicalin on cell apoptosis was studied following addition of either Baicalin or CQ (blocking autophagy) or Vitamin C (antioxidant) under HG conditions. As Baicalin administration attenuated cell apoptosis, suppressing ROS excessive production with Vitamin C could reduce the cell apoptosis, but attempts to block autophagy with CQ had no effect (Fig. 8). This suggests that massive ROS-induced cell autophagy plays an important role in cell apoptosis and that autophagy should exert a protective function on cell survival.

In consideration of maternal factors during application of Baicalin, STZ-induced diabetes mellitus mice were administered the compound. The results showed that Baicalin administration could attenuate diabetes mellitusinduced damage of kidney and liver to some extent; and successfully reduce the blood glucose levels of diabetic mice induced by STZ (Fig. 9) over the following weeks.

In summary (Fig. 10), Baicalin administration in mice could partially confront maternal hyperglycemia. Meanwhile, Baicalin could cross the placental barrier to protect cardiovascular formation from HG-induced damage. The exact mechanisms involved in reducing hyperglycemiaenhanced excessive ROS generation might involve suppression of outwardly rectifying $\mathrm{Cl}(-)$; unbalanced autophagy through regulating the p62 and subsequent cell apoptosis mainly induced by ROS. Therefore, embryonic angiodysplasia and heart tube malformation induced by hyperglycemia could be partially reduced through the intracellular homeostasis resulting from Baicalin administration, and Baicalin could be a potential candidate drug for gestational diabetes mellitus women. Further experimentations are required to investigate the therapeutic potential in treatment of diabetes and to determine the precise molecular mechanisms involved.

\section{Acknowledgements \\ This study was supported by the NSFC grant (81741016, 31771331), Science and Technology Planning Project of Guangdong Province (2014A020221091, 2017A020214015, 2017A050506029, 2016B030229002, 2014A020213008), Science and Technology Program of Guangzhou (201710010054), China Postdoctoral Science Foundation (2015T80940), Guangdong Natural Science Foundation (2016A030311044), The Fundamental Research Funds for the Central Universities (21617466), The Funds for Young Creative Talents of Higher Education in Guangdong Province (2014KQNCX026) and Research Grant of Key Laboratory of Regenerative Medicine, Ministry of Education, Jinan University (No. ZSYX-M-00001 and ZSYX-T-00001).}

\footnotetext{
Author details

${ }^{1}$ Division of Histology \& Embryology, Joint Laboratory for Embryonic Development \& Prenatal Medicine, Medical College, Jinan University, Guangzhou 510632, China. ${ }^{2}$ Chinese Medicine College, Jinan University, Guangzhou 510632, China. ${ }^{3}$ Key Laboratory for Regenerative Medicine of the Ministry of Education, Jinan University, Guangzhou 510632, China. ${ }^{4}$ Department of Pediatrics and Neonatology, Institute of Fetal-Preterm Labor Medicine; The First Affiliated Hospital, Jinan University, Guangzhou 510630, China. ${ }^{5}$ Norwich Medical School, University of East Anglia, Norwich, Norfolk, UK
}

${ }^{6}$ Division of Cell and Developmental Biology, University of Dundee, Dundee DD1 5EH, UK. 7Department of Pathophysiology, Institute of Brain Research, Medical College, Jinan University, Guangzhou 510632, China

\section{Authors' contributions}

G.W., J.L., L.G., Z.S., X.Z., G.L., Y.Y. and K.L. researched data. G.W., X.C., Y.B., M.C., L. C., D.L. and X.Y. contributed to discussion and edited manuscript. X.Y. wrote the manuscript.

\section{Conflict of interest}

The authors declare that they have no conflict of interest.

\section{Publisher's note}

Springer Nature remains neutral with regard to jurisdictional claims in published maps and institutional affiliations.

Supplementary Information accompanies this paper at https://doi.org/ 10.1038/s41419-018-0318-2

Received: 21 November 2017 Revised: 12 January 2018 Accepted: 16 January 2018

Published online: 14 February 2018

\section{References}

1. Tian, X. Y. et al. The effects of Scutellaria baicalensis extract on embryonic development in mice. Birth Defects Res. B Dev. Reprod. Toxicol. 86, 79-84 (2009).

2. Abel, E. L. Prenatal effects of alcohol. Drug Alcohol Depend. 14, 1-10 (1984).

3. Wang, P. et al. Baicalin alleviates ischemia-induced memory impairment by inhibiting the phosphorylation of CaMKIl in hippocampus. Brain Res. 1642, 95-103 (2016)

4. Wang, C. Z. et al. Colon cancer chemopreventive effects of baicalein, an active enteric microbiome metabolite from baicalin. Int. J. Oncol. 47, 1749-1758 (2015).

5. Wang, F., Xu, Z., Ren, L., Tsang, S. Y. \& Xue, H. GABA A receptor subtype selectivity underlying selective anxiolytic effect of baicalin. Neuropharmacology 55, 1231-1237 (2008).

6. Baek, J. S. et al. Comparative pharmacokinetics of a marker compound, baicalin in KOB extract after oral administration to normal and allergic-induced rats. Drug Deliv. 21, 453-458 (2014).

7. Zhang, Y. M. et al. Baicalin promotes embryo adhesion and implantation by upregulating fucosyltransferase IV (FUT4) via Wnt/beta-catenin signaling pathway. FEBS Lett. 589, 1225-1233 (2015).

8. Yin, F. et al. Baicalin prevents the production of hydrogen peroxide and oxidative stress induced by Abeta aggregation in SH-SY5Y cells. Neurosci. Lett. 492, 76-79 (2011)

9. Qi, X. et al. Baicalin increases developmental competence of mouse embryos in vitro by inhibiting cellular apoptosis and modulating HSP70 and DNMT expression. J. Reprod. Dev. 62, 561-569 (2016).

10. Yutzey, K. E. \& Kirby, M. L. Wherefore heart thou? Embryonic origins of cardiogenic mesoderm. Dev. Dyn. 223, 307-320 (2002).

11. Linask, K. K. Regulation of heart morphology: current molecular and cellular perspectives on the coordinated emergence of cardiac form and function. Birth Defects Res. C Embryo Today 69, 14-24 (2003).

12. Schultheiss, T. M., Xydas, S. \& Lassar, A. B. Induction of avian cardiac myogenesis by anterior endoderm. Development 121, 4203-4214 (1995).

13. Nakajima, Y. Cardiovascular development: structure and molecular mechanism. Anat. Sci. Int. 84, 65-66 (2009).

14. Nakajima, Y. et al. Heart development before beating. Anat. Sci. Int. 84, 67-76 (2009).

15. Brand, T. Heart development: molecular insights into cardiac specification and early morphogenesis. Dev. Biol. 258, 1-19 (2003).

16. Patan, S. Vasculogenesis and angiogenesis. Cancer Treat. Res. 117, 3-32 (2004).

17. Li, Y. et al. PTEN is involved in modulation of vasculogenesis in early chick embryos. Biol. Open 2, 587-595 (2013).

18. Li, Y. et al. Excess ROS induced by AAPH causes myocardial hypertrophy in the developing chick embryo. Int. J. Cardiol. 176, 62-73 (2014). 
19. Wang, G. et al. Angiogenesis is repressed by ethanol exposure during chick embryonic development. J. Appl. Toxicol. 36, 692-701 (2016).

20. He, Y. Q. et al. Dimethyl phenyl piperazine iodide (DMPP) induces glioma regression by inhibiting angiogenesis. Exp. Cell Res. 320, 354-364 (2014).

21. Ejdesjo, A., Wentzel, P. \& Eriksson, U. J. Influence of maternal metabolism and parental genetics on fetal maldevelopment in diabetic rat pregnancy. Am. J. Physiol. Endocrinol. Metab. 302, E1198-E1209 (2012).

22. Wang, $G$. et al. Autophagy is involved in high glucose-induced heart tube malformation. Cell Cycle 14, 772-783 (2015).

23. Jin, Y. M. et al. High glucose level induces cardiovascular dysplasia during early embryo development. Exp. Clin. Endocrinol. Diabetes 121, 448-454 (2013).

24. Chen, J. G. et al. Baicalin can attenuate the inhibitory effects of mifepristone on Wnt pathway during peri-implantation period in mice. J Steroid Biochem Mol Biol 149, 11-16 (2015)

25. Hamburger, V. \& Hamilton, H. L. A series of normal stages in the development of the chick embryo. 1951. Dev. Dyn. 195, 231-272 (1992).

26. Chapman, S. C., Collignon, J., Schoenwolf, G. C. \& Lumsden, A. Improved method for chick whole-embryo culture using a filter paper carrier. Dev. Dyn. 220, 284-289 (2001)

27. Cheng, $X$. et al. Effects of 2,5-hexanedione on angiogenesis and vasculogenesis in chick embryos. Reprod. Toxicol. 51, 79-89 (2015).

28. Henrique, D. et al. Expression of a Delta homologue in prospective neurons in the chick. Nature 375, 787-790 (1995).

29. Maroto, M. et al. Ectopic Pax-3 activates MyoD and Myf-5 expression in embryonic mesoderm and neural tissue. Cell 89, 139-148 (1997).

30. Dugaiczyk, A. et al. Cloning and sequencing of a deoxyribonucleic acid copy of glyceraldehyde-3-phosphate dehydrogenase messenger ribonucleic acid isolated from chicken muscle. Biochemistry 22, 1605-1613 (1983).

31. Khanal, T. et al. Protective role of intestinal bacterial metabolism against baicalin-induced toxicity in HepG2 cell cultures. J. Toxicol. Sci. 37, 363-371 (2012).

32. Jin, $Y$. et al. Effects of oxidative stress on hyperglycaemia-induced brain malformations in a diabetes mouse model. Exp. Cell Res. 347, 201-211 (2016)

33. Noh, K. et al. Role of intestinal microbiota in baicalin-induced drug interaction and its pharmacokinetics. Molecules 21, 337 (2016).

34. Kang, M. J. et al. Role of metabolism by intestinal microbiota in pharmacokinetics of oral baicalin. Arch. Pharm. Res. 37, 371-378 (2014).

35. Ishimaru, K. et al. Two flavone 2'-glucosides from Scutellaria baicalensis. Phytochemistry 40, 279-281 (1995).
36. Lin, C. C. \& Shieh, D. E. The anti-inflammatory activity of Scutellaria rivularis extracts and its active components, baicalin, baicalein and wogonin. Am. J. Chin. Med. 24, 31-36 (1996).

37. Li-Weber, M. New therapeutic aspects of flavones: the anticancer properties of Scutellaria and its main active constituents Wogonin, Baicalein and Baicalin. Cancer Treat. Rev. 35, 57-68 (2009).

38. Trinh, H. T., Joh, E. H., Kwak, H. Y., Baek, N. I. \& Kim, D. H. Anti-pruritic effect of baicalin and its metabolites, baicalein and oroxylin A, in mice. Acta Pharmacol. Sin. 31, 718-724 (2010).

39. Tufan, A. C. \& Satiroglu-Tufan, N. L. The chick embryo chorioallantoic membrane as a model system for the study of tumor angiogenesis, invasion and development of anti-angiogenic agents. Curr. Cancer Drug Targets 5, 249-266 (2005).

40. Song, D. et al. [Ingredients of Shuanghuanglian injection powder permeation through placental barrier of rat in pregnancy]. Zhongguo Zhong Yao Za Zhi 35, 1626-1629 (2010).

41. Haribalaganesh, R., Sheikpranbabu, S., Elayappan, B., Venkataraman, D. \& Gurunathan, S. Pigment-epithelium-derived factor down regulates hyperglycemia-induced apoptosis via PI3K/Akt activation in goat retinal pericytes. Angiogenesis 12, 381-389 (2009).

42. Han, S. S. et al. Investigating the mechanism of hyperglycemia-induced fetal cardiac hypertrophy. PLOS ONE 10, e0139141 (2015).

43. Maeno, E., Ishizaki, Y., Kanaseki, T., Hazama, A. \& Okada, Y. Normotonic cell shrinkage because of disordered volume regulation is an early prerequisite to apoptosis. Proc. Natl. Acad. Sci. USA 97, 9487-9492 (2000).

44. Bortner, C. D. \& Cidlowski, J. A. Cell shrinkage and monovalent cation fluxes: role in apoptosis. Arch. Biochem. Biophys. 462, 176-188 (2007).

45. Hasegawa, Y., Shimizu, T., Takahashi, N. \& Okada, Y. The apoptotic volume decrease is an upstream event of MAP kinase activation during Staurosporine-induced apoptosis in HeLa cells. Int J. Mol. Sci. 13, 9363-9379 (2012).

46. de Oliveira, M. R. et al. The effects of baicalein and baicalin on mitochondrial function and dynamics: a review. Pharmacol. Res. 100, 296-308 (2015).

47. Li, C. \& Siragy, H. M. (Pro)renin receptor regulates autophagy and apoptosis in podocytes exposed to high glucose. Am. J. Physiol. Endocrinol. Metab. 309, E302-E310 (2015).

48. $\mathrm{Xu}$, L. et al. Ursolic acid improves podocyte injury caused by high glucose. Nephrol. Dial. Transplant. 32, 1285-1293 (2017). 